\section{SPIN IN THE UNIVERSE}

$\mathrm{S}$ R EDMUND WHITTAKER discussed "The Spin of the Universe" in his presidential address to the Royal Society of Edinburgh at the annual statutory meeting held on October 23, 1944 (Yeur Book of the Royal Nociety of Edinburgh, 1945.

Starting with Earl Rosse's discoveries of spiral nebulæ, the first of which was $M 51$ (although examined previously by several astronomers the much greater resolving power used by Lord Rosse showed its spiral character for the first time), Whittaker proceeds to examine the cosmological significance of rotation and spin characteristics of the spiral nebulæ. $\mathrm{He}$ refers to the theory propounded by $\mathrm{H}$. Alfvén (Ark. Mat., Astr., och. Fys., 28 A, No. 6; 1942), which accounts for the angular momentum possessed by the planets of the solar system (a short outline of this theory appeared in Nature, 150, 405, Oct. 3, 1942, and 152, 721, Dec. 18, 1943). Alfvén's theory would apply to the stars as well as to the sun, and would lead us to assume that a star is generally accompanied by a family of planets - a view corroborated by recent discoveries of planets associated with other stars. His theory shows how spin can be acquired by material bodies under the action of electric and magnetic fields, or, in other words, spin can be transferred from ether to matter, and hence it is natural to inquire whether anything is known about spin or angular momentum in the ether.

Since the discovery of the theory of relativity, the ether has been discarded except as the name of an invisible transmitter of actions at a distance, and the ether theories have been replaced by the new science of atomic physies. How has the change, in which the fundamental entities are elementary wave-particles or corporundals of certain kinds, affected our conception of invisible spin? Within the last two decades it has been found that each of these elementary particles possesses a certain definite quantity of spin, and this is of great importance in determining the behaviour of the particle. A short description of quantum numbers is followed by an account of the discovery in 1925 that a third quantum number is required to describe an electronic level, a discovery which immediately led to the question, "To what physical character of the atom does this new third quantum number correspond." Reasons were soon fortheoming to suggest that this third number corresponded to the total spin of the whole atom, just as the second quantum number represented the angular momentum or spin of the orbital motion of the electron, and it was shown that it was not the spin of the nucleus but of the electron which took place.

A study of the Zeeman effect led to the conclusion that the angular momentum or spin of the electron is $\frac{1}{2} h$, where $h$ is Planck's constant of action divided by $2 \pi$, and that the electron has a certain fixed magnetic moment associated with the spin. It is impossible to verify the spin and magnetic moment by direct observation, owing to the uncertainty principle, and the phenomenon of electron-spin belongs to quantum theory, for which reason it possesses peculiarities not met with in classical dynamics. It is a quantum-mechanical entity, and when we speak of its component in the direction of the $z$-axis, say, the only meaning attached to the statement is that there is a certain probability that the axis of spin will be parallel to the $z$-axis and the spin-component of amount $\frac{1}{2} h$, and also the probability that the axis of spin will be antiparallel to the $z$-axis, the spin component being now $-\frac{1}{2} h$. It has boen found that each of the other elementary particles also possesses a definite amount of spin; the proton, the neutron and the positron can each have the same spin as the electron, $\frac{1}{2} h$, while the photon or light corpuscle has the spin $h$, and the spin of the meson may have either of the values 0 or $h$.

Whittaker refers to the important part that the spin of the elementary particle plays in chemical combination and to its influence on what is called the statistical behaviour of the elementary particles, and deals briefly with the Maxwellian statistics, the Fermi statistics, and the Bose statistics, generally recognized under the composite titles, Maxwell-Boltzman, FermiDirac and Bose-Einstein, but it is impossible in a limited space to deal with every feature of the address. One important matter may be mentioned. The correlation between the particle and the wave properties of the elementary particles has been long known, so far as concerns energy, momentum and velocity, and the question arises, "What feature of the waves is to be interpreted as spin ?" Sir Charles Darwin discovered the true solution in 1927, and concluded that the wave-aspect of spin was simply the capacity to exist in dif erent states possessing the same energy and momentum, analogous to diferently polarized waves of light. In 1928 Dirac found the wave equation of the electron in a form which was invariant under all the transformations associated with the principle of relativity, and spin appears to be a consequence of the symmetry of the cosmos with respect to space and time. The concept of spin thus emerged in a new light; it had originated in atomic physics, and it was now lifted into a new context, that of relativity theory.

Relativists had directed their attention for many years to the remarkable difference existing, as regards relativity, between motions of translation and of rotation. According to the principle of relativity, it is impossible by observational means to detect uniform motion of translation which occurs wholly within the system; but the case is quite different as regards rotation. We can speak of velocities of rotation in an absolute manner, without specifying the framework with respect to which the angular displacement takes place. Certain problems in the relativity theory of spin arise, to which reference is made, and some of these are still controversial. As Whittaker points out, the study of rotation is essentially a cosmic problem, and in attempting to solve it we are brought back to the spiral nebulæ. It seems probable that their inner regions rotate as if rigidly connected, the linear velocity increasing with the distance, while in the outer parts the linear velocity decreases with increasing distance. Prof. E. A. Milne has proposed an explanation of the rotation of the spiral nebulæ and of the possession of spin by most large objects and groups of objects in the universe. He has deduced from his kinematical relativity that the angular momentum of any cosmical system increases proportionally to the time, that is, to what he calls kinematic time, which is time as measured by atomic vibrations and radioactive clocks. Hence a spin which was initially small will grow continually greater, and there is therefore nothing surprising in the existence of large accumulations of angular momentum. No theory to explain spin in the universe has yet been generally accepted, but it is reasonable to hope for a solution of this fundamental problem of cosmology in the near future. 